\title{
Increased prevalence of autoimmune disease in patients with unilateral compared with bilateral moyamoya disease
}

\author{
Jian-Bin Chen, MD, Yi Liu, MD, Liang-Xue Zhou, MD, Hong Sun, MD, Min He, MD, and \\ Chao You, MD, PhD
}

Department of Neurosurgery, West China Hospital, West China School of Medicine, Sichuan University, Chengdu, Sichuan Province, People's Republic of China

\begin{abstract}
OBJECTIVE This study explored whether there were differences between the autoimmune disease prevalence rates in unilateral and bilateral moyamoya disease (MMD).

METHODS The authors performed a retrospective review of data obtained from the medical records of their hospital, analyzing and comparing the clinical characteristics and prevalence rates of all autoimmune diseases that were associated with unilateral and bilateral MMD in their hospital from January 1995 to October 2014.

RESULTS Three hundred sixteen patients with bilateral MMD and 68 with unilateral MMD were identified. The results indicated that patients with unilateral MMD were more likely to be female than were patients with bilateral MMD $(67.6 \%$ vs $51.3 \%, p=0.014$, odds ratio [OR] 1.99). Overall, non-autoimmune comorbidities tended to be more prevalent in the unilateral MMD cases than in the bilateral MMD cases ( $17.6 \%$ vs $9.8 \%, p=0.063$, OR 1.97, chi-square test). Autoimmune thyroid disease and other autoimmune diseases also tended to be more prevalent in the unilateral MMD cases than in the bilateral MMD cases (19.1\% vs 10.8\%, $p=0.056$, OR 1.96 and $8.8 \%$ vs $3.5 \%, p=0.092$, OR 2.77, respectively, chi-square test). The overall autoimmune disease prevalence in the unilateral MMD cases was significantly higher than in the bilateral MMD cases $(26.5 \%$ vs $13.6 \%, p=0.008$, OR $2.29,95 \% \mathrm{Cl} 1.22-4.28$, chi-square test). Multiple logistic regression analysis showed that autoimmune disease was more likely to be associated with unilateral than with bilateral MMD ( $p=0.039$, OR 10.91, 95\% Cl 1.13-105.25).
\end{abstract}

CONCLUSIONS This study indicated a higher overall autoimmune disease prevalence in unilateral than in bilateral MMD. Unilateral MMD may be more associated with autoimmune disease than bilateral MMD. Different pathogenetic mechanisms may underlie moyamoya vessel formation in unilateral and bilateral MMD.

http://thejns.org/doi/abs/10.3171/2015.4.JNS142936

KEY WORDS unilateral; bilateral; moyamoya; autoimmune; prevalence; pathogenesis; vascular disorders

$\mathrm{M}$ OYAMOYA vasculopathy (MMV) is a disorder that is characterized by stenosis or occlusion of the terminal internal carotid artery (ICA) and the proximal portion of the anterior and/or middle cerebral arteries. Additionally, it is accompanied by net-like collateral vessel formations, which are termed the "moyamoya" vessels..$^{14,18}$ Moyamoya disease (MMD) is defined by the presence of bilateral MMV with an unknown etiology or the presence of unilateral MMV at 1 side with obvious terminal contralateral ICA stenosis. ${ }^{4}$ Unilateral MMD is defined by typical angiographic evidence of MMD unilaterally with normal or equivocal contralateral findings. ${ }^{4}$ The concept that unilateral MMD is an early form of bilateral MMD remains controversial, and the pathogenesis of moyamoya disorders is not fully understood. . $, 11,14,18^{2}$

MMD has been reportedly associated with various entities. ${ }^{2,8,9,13,16,24}$ Analysis of the coexisting diseases that occur with moyamoya may be helpful in exploring moyamoya pathogeneses. Several reports have noted MMD in association with autoimmune diseases, including autoim-

ABBREVIATIONS AIG = autoimmune gastritis; APAS = antiphospholipid antibody syndrome; $A P S$ = autoimmune polyendocrine syndrome; GD = Graves' disease; $H$ T = Hashimoto thyroiditis; ICA = internal carotid artery; ICD-10 = International Classification of Diseases, 10th Revision; MCA = middle cerebral artery; MMD = moyamoya disease; $\mathrm{MMV}=$ moyamoya vasculopathy; $\mathrm{PBC}=$ primary biliary cirrhosis; $\mathrm{SLE}=$ systemic lupus erythematosus; $\mathrm{TIA}=$ transient ischemic attack.

SUBMITTED December 26, 2014. ACCEPTED April 6, 2015.

INCLUDE WHEN CITING Published online September 25, 2015; DOI: 10.3171/2015.4.JNS142936. 
mune thyroid disease, Type 1 diabetes mellitus, and systemic lupus erythematosus (SLE) ${ }^{2,13,16,24}$ Recently, the autoimmune disease prevalence in bilateral MMD was also reported in a midwestern US population. ${ }^{2}$ However, the clinical characteristics of autoimmune disease in unilateral MMD have not been reported in the literature. Whether there are differences between the autoimmune associations that occur with unilateral and bilateral disease has not been investigated. We therefore sought to explore the clinical characteristics of autoimmune disease in unilateral and bilateral MMD to gain insight into the potential differences between the autoimmune associations of these 2 subgroups in this retrospective study.

\section{Methods}

\section{Patient Eligibility and Selection}

This study was a retrospective chart review of cases involving patients seen between January 1995 and October 2014 at West China Hospital of Sichuan University. Patients were identified for potential inclusion based on the ICD-9 code 4375 (MMD). In order to qualify for inclusion in the study, patients had to have angiographically identified unilateral or bilateral MMD. Patients with intracranial atherosclerosis of angiographic evidence, reversible vasoconstriction syndrome, intracranial dissection, systemic vasculitis, meningitis, head injury, previous cranial base radiation therapy, or undefined inflammatory processes were excluded.

A retrospective chart review was performed on cases involving patients who met the inclusion criteria. This study was approved by the Sichuan University ethics committee. Vascular images, including digital subtraction, $\mathrm{MR}$, and CT angiography studies, were reviewed in detail by 2 neurosurgeons (C.Y. and J.B.C.) and 1 neuroradiologist (Y.L.). The MMD diagnoses were based on the Japanese Ministry of Health and Welfare Research Committee on Spontaneous Occlusion of the Circle of Willis (MMD) guidelines, which were established in 1997.4 "Bilateral MMD" is a bilateral ICA terminal steno-occlusion with bilateral moyamoya vessel development. ${ }^{5,6}$ "Unilateral MMD" is a unilateral ICA terminal steno-occlusion with moyamoya vessel development., ${ }^{5,6}$ The collected data included race/ethnicity (based on patient self-report), sex, age at presentation, clinical presentations, angiographic findings (laterality and involved arteries), relevant family history, accepted stroke risk factors at initial presentation, and other comorbidities-specifically, syndromes with a known association with moyamoya and all autoimmune diseases (Table 1). Autoimmune disease was assessed, and thyroid disease was confirmed as autoimmune in nature by confirming that there was no other clear cause.

\section{Clinical Characteristic Assessments}

The patients' basic clinical characteristics were reviewed and analyzed (Table 2). We compared the demographic characteristics (e.g., age, sex, and race/ethnicity), initial presentations (e.g., transient ischemic attack [TIA], headache, cognitive decline, memory loss, dizziness, motor weakness, aphasia, seizure, asymptomatic, and others), and results of imaging examinations (CT/MRI) between the patients with unilateral MMD and those with bilateral MMD.

\section{Comparison of All Autoimmune Diseases, Other Comorbidities, and Cerebrovascular Risk Factors}

The autoimmune disease and other comorbidity prevalence rates were analyzed and compared between the 2 groups of patients. All of the following autoimmune diseases were considered in the study: autoimmune thyroid diseases, including Graves' disease (GD) and Hashimoto thyroiditis (HT); autoimmune Type 1 diabetes mellitus; and other autoimmune diseases, including autoimmune gastritis (AIG), systemic lupus erythematosus (SLE), antiphospholipid antibody syndrome (APAS), autoimmune polyendocrine syndrome (APS), and primary biliary cirrhosis (PBC). All autoimmune diseases and other comorbidities were evaluated with medical testing, and specific diagnoses were made according to the International Classification of Diseases, 10th Revision (ICD-10). Additionally, the prevalence rates of cerebrovascular risk factors, including hyperlipidemia, hypertension, history of tobacco use, Type 2 diabetes mellitus, and fibromuscular dysplasia, were compared between the unilateral and bilateral MMD groups (Table 1).

\section{Statistical Analysis}

Statistical analyses were performed with SPSS 18.0 (SPSS Inc.). The categorical variables were expressed as percentages and analyzed with the chi-square test, and the continuous variables were expressed as mean \pm standard deviation and compared with the Student t-test. Differences were considered to be significant with a $\mathrm{p}$ value $<0.05$. Additionally, we performed multivariate logistic regression analysis to identify whether each autoimmune disease was more likely to be associated with unilateral or bilateral MMD after controlling for demographic characteristics (e.g., age and sex), risk factors, overall autoimmune diseases, and other comorbidities. Variables with $\mathrm{p}$ $<0.15$ on univariate analysis were considered to represent explanatory variables.

\section{Results}

\section{Clinical Characteristics of the Patient Groups}

The patients' clinical characteristics are presented in Table 2. Three hundred sixteen patients with bilateral MMD and 68 patients with unilateral MMD met the study criteria. Patients with unilateral MMD were more likely to be female than patients with bilateral MMD (67.6\% vs $51.3 \%, p=0.014$; odds ratio [OR] 1.99; 95\% confidence interval [CI] 1.14-3.46, chi-square test). There was no significant difference between the unilateral and bilateral cases with respect to family history of MMD (present in 5 [7.4\%] of 68 cases of unilateral MMD and 16 [5.1\%] of 316 cases of bilateral MMD, $p=0.646$ ). There was also no significant difference between the 2 groups with respect to age at presentation, TIA, headache, cognitive decline, memory loss, dizziness, motor weakness, aphasia, seizure, or asymptomatic presentation ( $\mathrm{p}>0.5$ for all, chi-square test). The races/ethnicities of patients with unilateral MMD included: Han Chinese (91.2\%), Yi (2.9\%), Zang (1.5\%), 
TABLE 1. Comparison of the total autoimmune disease and non-autoimmune comorbidity prevalence rates between patients with unilateral and bilateral MMD*

\begin{tabular}{|c|c|c|c|c|}
\hline Variable & Unilateral MMD $(n=68)$ & Bilateral MMD $(n=316)$ & p Value & OR $[95 \% \mathrm{Cl}]$ \\
\hline Female & $46(67.6)$ & $162(51.3)$ & 0.014 & $1.99[1.14-3.46]$ \\
\hline Age at onset in yrs (mean \pm SD) & $38.9 \pm 11.5$ & $37.3 \pm 13.7$ & $>0.05$ & \\
\hline Family history & $5(7.4)$ & $16(5.1)$ & 0.646 & $1.49[0.53-4.21]$ \\
\hline \multicolumn{5}{|l|}{ Risk factors } \\
\hline Tobacco use & $12(17.6)$ & $52(16.5)$ & 0.811 & $1.09[0.55-2.17]$ \\
\hline Hypertension & $14(20.6)$ & $69(21.8)$ & 0.821 & $0.93[0.49-1.77]$ \\
\hline Hyperlipidemia & $18(26.5)$ & $58(18.4)$ & 0.128 & $1.60[0.87-2.95]$ \\
\hline Type 2 diabetes mellitus & $6(8.8)$ & $33(10.4)$ & 0.688 & $0.83[0.33-2.07]$ \\
\hline Autoimmune Type 1 diabetes mellitus & $5(7.4)$ & $13(4.1)$ & 0.406 & $1.85[0.64-5.37]$ \\
\hline Autoimmune thyroid disease & $13(19.1)$ & $34(10.8)$ & 0.056 & $1.96[0.97-3.95]$ \\
\hline Graves' disease & $8(11.8)$ & $21(6.6)$ & 0.147 & $1.87[0.79-4.43]$ \\
\hline Hashimoto thyroiditis & $5(7.4)$ & $13(4.1)$ & 0.406 & $1.85[0.64-5.37]$ \\
\hline Autoimmune disease of any other kind & $6(8.8)$ & $11(3.5)$ & 0.092 & $2.77[0.99-7.77]$ \\
\hline AIG & $1(1.47)$ & $1(0.32)$ & 0.323 & $4.70[0.29-76.11]$ \\
\hline SLE & $1(1.47)$ & $3(0.95)$ & 0.543 & $1.56[0.16-15.20]$ \\
\hline APAS & $3(4.4)$ & $5(1.58)$ & 0.153 & $2.87[0.67-12.31]$ \\
\hline APS & $1(1.47)$ & $1(0.32)$ & 0.323 & $4.70[0.29-76.11]$ \\
\hline PBC & $0(0)$ & $1(0.32)$ & 1.00 & $1.00[0.997-1.01]$ \\
\hline Overall autoimmune diseases & $18(26.5)$ & $43(13.6)$ & 0.008 & $2.29[1.22-4.28]$ \\
\hline Other comorbidities & $12(17.6)$ & $31(9.8)$ & 0.063 & $1.97[0.95-4.07]$ \\
\hline NF-1 & 2 & 4 & 0.288 & $2.36[0.42-13.17]$ \\
\hline ADPKD & 1 & 2 & 0.444 & $2.34[0.21-26.22]$ \\
\hline Homocysteinemia & 2 & 4 & 0.467 & $2.36[0.42-13.17]$ \\
\hline Fibrinogenemia & 1 & 3 & 0.543 & $1.56[0.16-15.20]$ \\
\hline Protein S deficiency & 1 & 4 & 1.000 & $1.16[0.13-10.58]$ \\
\hline Antithrombin III deficiency & 1 & 4 & 1.000 & $1.16[0.13-10.58]$ \\
\hline Down syndrome & 4 & 10 & 0.288 & $1.91[0.58-6.29]$ \\
\hline
\end{tabular}

Qiang (1.5\%), and others (2.9\%). The race/ethnicity component analysis showed no significant differences between the unilateral and bilateral MMD groups. In the unilateral group, the imaging examinations (CT/MRI) revealed that the incidence of hemorrhage was significantly higher than that of ischemic stroke $(58.8 \%$ vs $30.9 \%, \mathrm{p}=0.001$, chisquare test). However, the imaging outcomes (ischemia and hemorrhage) did not differ significantly between the unilateral and bilateral MMD groups (30.9\% vs 33.5\%, $\mathrm{p}=0.672$ and $58.8 \%$ vs $51.9 \%, \mathrm{p}=0.299$, respectively, chi-square test). The incidence of coexisting intracranial aneurysms also did not differ significantly between the 2 groups $(1.5 \%$ vs $0.9 \%, \mathrm{p}=0.543$, chi-square test).

\section{Anatomical Features of Unilateral MMD}

Multiple anterior circulation arteries were involved in $69.1 \%$ of patients with unilateral MMD, with $14.7 \%$ of these patients having isolated ICA involvement and 7.4\% of patients having isolated middle cerebral artery (MCA) involvement. The moyamoya vessels extended to the posterior circulation in $8.8 \%$ of the patients with unilateral MMD (Table 2).

\section{Cerebrovascular Risk Factors}

We observed the following prevalence rates for patients with unilateral MMD: tobacco use, $17.6 \%$ (12/68); hypertension, 20.6\% (21/68); hyperlipidemia, 26.5\% (18/68); and Type 2 diabetes mellitus, 6.8\% (6/68). The cerebrovascular risk factors were not more frequent in the unilateral MMD group than in the bilateral MMD group ( $p>0.05$ for all, chi-square test, Table 1).

\section{Clinical Characteristics of Patients with Non-Autoimmune Comorbidities}

The following nonautoimmune comorbidities were observed in the unilateral disease patients: neurofibromatosis Type 1 (2 patients), autosomal dominant polycystic kidney disease (1 patient), homocysteinemia (4 patients), fibrino- 
TABLE 2. Comparison of demographic and clinical characteristics and manifestations in unilateral and bilateral MMD cases

\begin{tabular}{|c|c|c|c|}
\hline Variable & $\begin{array}{c}\text { Unilateral } \\
\text { MMD }(n=68)\end{array}$ & $\begin{array}{l}\text { Bilateral MMD } \\
\quad(n=316)\end{array}$ & $\begin{array}{c}p \\
\text { Value }\end{array}$ \\
\hline \multicolumn{4}{|l|}{ Race/ethnicity } \\
\hline Han & $62(91.2)$ & $290(91.8)$ & 0.872 \\
\hline Yi & $2(2.9)$ & $8(2.5)$ & 1 \\
\hline Zang & $1(1.5)$ & $4(1.3)$ & 1 \\
\hline Qiang & $1(1.5)$ & $3(0.95)$ & 0.543 \\
\hline Other & $2(2.9)$ & $7(2.2)$ & 1 \\
\hline \multicolumn{4}{|l|}{ Initial presentation } \\
\hline TIA & $19(27.9)$ & $69(21.8)$ & 0.277 \\
\hline Headache & $10(14.7)$ & $41(13.0)$ & 0.703 \\
\hline Cognitive decline & $4(5.9)$ & $16(5.1)$ & 1 \\
\hline Memory loss & $5(7.4)$ & $30(9.5)$ & 0.578 \\
\hline Dizziness & $4(5.9)$ & $14(4.4)$ & 0.843 \\
\hline Motor weakness & $10(14.7)$ & $40(12.7)$ & 0.649 \\
\hline Aphasia & $3(4.4)$ & $15(4.7)$ & 1 \\
\hline Seizure & $4(5.9)$ & $16(5.1)$ & 1 \\
\hline Asymptomatic & $9(13.2)$ & $75(23.7)$ & 0.057 \\
\hline \multicolumn{4}{|l|}{ Imaging findings (CT or MRI) } \\
\hline Ischemic stroke & $21(30.9)$ & $106(33.5)$ & 0.672 \\
\hline Hemorrhage & $40(58.8)$ & $164(51.9)$ & 0.299 \\
\hline Normal & $7(10.3)$ & $46(13.3)$ & 0.355 \\
\hline Aneurysm & $1(1.5)$ & $3(0.9)$ & 0.543 \\
\hline \multicolumn{4}{|l|}{ Location } \\
\hline ICA alone & $10(14.7)$ & & \\
\hline MCA alone & $5(7.4)$ & & \\
\hline Multiple locations & $47(69.1)$ & & \\
\hline Pst circulation involvement & $6(8.8)$ & & \\
\hline \multicolumn{4}{|l|}{ Side } \\
\hline Right & $36(52.9)$ & & \\
\hline Left & $32(47.1)$ & & \\
\hline
\end{tabular}

genemia (1 patient), protein $S$ deficiency (1 patient), antithrombin III deficiency (1 patient), and Down syndrome (2 patients). The prevalence of each comorbidity type was not significantly higher in the unilateral than in the bilateral moyamoya patients ( $\mathrm{p}>0.05$ for all, chi-square test, Table 1). However, the nonautoimmune comorbidity prevalence rates tended to be higher in the unilateral than in the bilateral moyamoya patients $(17.6 \%$ vs $9.8 \%, p=0.063$, OR $1.97,95 \%$ CI $0.95-4.07$, chi-square test, Table 1).

\section{Autoimmune Disease Comparison Between Unilateral and Bilateral MMD Cases}

The autoimmune disease prevalence rates are presented in Table 1. We found that the prevalence of autoimmune Type 1 diabetes mellitus did not differ significantly between the unilateral and bilateral MMD groups $(7.4 \%$ vs $4.1 \%, p=0.406$, chi-square test). Although there was no statistically significant differences, the prevalence rates for autoimmune thyroid disease and all of the other autoim- mune diseases tended to be higher in the unilateral MMD group than in the bilateral MMD group (19.1\% vs $10.8 \%$, $\mathrm{p}=0.056$, OR $1.96 ; 8.8 \%$ vs $3.5 \%, \mathrm{p}=0.092$, OR 2.77 ; chi-square test). With respect to autoimmune thyroid disease subtypes, the GD and HT prevalence rates were not significantly different in the unilateral and bilateral MMD groups $(\mathrm{GD}, 11.8 \%$ vs $6.6 \%, \mathrm{p}=0.147$; HT, $7.4 \%$ vs $4.1 \%$, $\mathrm{p}$ $=0.406$; chi-square test). The other autoimmune diseases types in the unilateral MMD group included the following: AIG (1 patient), SLE (1 patient), APAS (3 patients), and APS (1 patient). The other autoimmune disease types in the bilateral MMD group included following: AIG (1 patient), SLE (3 patients), APAS (5 patients), APS (1 patient), and PBC (1 patient). The prevalence of the other autoimmune disorders did not differ significantly between the 2 groups ( $p>0.5$ for all, chi-square test). One patient with unilateral MMD had 2 types of autoimmune disease, and 2 patients with bilateral MMD had 2 autoimmune disease types. The overall prevalence of autoimmune disease was significantly higher in the unilateral MMD group than in the bilateral MMD group (26.5\% vs $13.6 \%$, $\mathrm{p}=$ 0.008 , OR $2.29,95 \%$ CI 1.22-4.28, chi-square test).

Multiple logistic regression analysis was conducted to further evaluate the differences in prevalence of the autoimmune diseases that were associated with the unilateral and bilateral MMD cases. After adjusting for covariates, including sex, age, Type 2 diabetes mellitus, risk factors, overall autoimmune disease, and other comorbidities, autoimmune disease was more likely to be associated with unilateral MMD than bilateral MMD ( $\mathrm{p}=0.039$, OR 10.91, 95\% CI 1.13-105.25).

\section{Discussion}

In recent years, coexistence of other diseases with MMD has been increasingly reported, $, 2,8,9,13,16,24$ and some of these reports have specifically involved autoimmune disease. ${ }^{2,13,16,24}$ However, no previous studies have explored the autoimmune disease characteristics in patients with unilateral MMD in comparison with those in patients with bilateral MMD. Whether there were differences between the autoimmune associations that occurred with unilateral and bilateral moyamoya disorders remained unclear. The results of our study show that the overall autoimmune disease prevalence was significantly higher in the patients with unilateral MMD than in those with bilateral MMD. The nonautoimmune comorbidity prevalence rates also tended to be higher in the unilateral MMD group than in the bilateral MMD group. Additionally, the female-tomale ratio was 2.1:1 in the unilateral MMD group, and patients with unilateral MMD were more likely to be female than were patients with bilateral MMD. The multivariate analysis indicated that unilateral MMD was more likely to be associated with autoimmune disease than bilateral MMD.

The female-to-male ratio (2.1:1) in the unilateral MMD group was similar to ratios found in prior studies, which reported that the female-to-male ratio was approximately twofold in patients with unilateral disease., ${ }^{5,6,14,18}$ The proportion of female patients in the unilateral MMD group was also similar to that in previous reports, which showed 
a female predominance in Japan, South Korea, China, and the United States. In these reports, the female-to-male MMD patient ratio ranged from 1.72:1 to 2.5:1.,5,6,11,21 However, the female-to-male ratio was approximately 1:1 in our bilateral MMD group, which was similar to that in other Chinese cohort studies.,16 The results suggest that a sex distribution difference exists between unilateral and bilateral MMD cases, and that female preponderance may be greater in unilateral MMD than in bilateral disease. ${ }^{14,18,21}$ One previous study, which also analyzed the sex distribution, also indicated that unilateral MMD might be influenced by acquired diseases, including autoimmune disease. $^{5}$

The unilateral MMD clinical manifestations were divided into focal signs and nonspecific symptoms, such as headache and TIA. In this study, only $13.2 \%$ of the unilateral MMD patients had nonspecific symptoms or were asymptomatic, and there tended to be a lower proportion of asymptomatic cases in the unilateral group than in the bilateral group. These findings suggest that unilateral MMD patients tend to be more symptomatic, which is consistent with previous reports. ${ }^{6,10,12}$ The higher prevalence of concurrent disease in the unilateral MMD patients than in the bilateral MMD patients may suggest symptomatic differences between these 2 subgroups.

Previous studies have indicated that unilateral MMD tends to be observed with comorbidities. ${ }^{12,14,17,18}$ In this study, the prevalence of the individual comorbidity types was not significantly greater in unilateral cases than in bilateral cases. However, the overall prevalence rates of comorbidities tended to be higher in the unilateral MMD patients than in the bilateral MMD group. These results suggest that coexisting diseases may be involved in both unilateral and bilateral moyamoya disorders.

Previous studies have demonstrated the association between MMD and autoimmune disease, namely, that there is an increased prevalence of autoimmune disease in patients with MMD. Additionally, the authors of these studies hypothesized that there is a potential autoimmune component to the pathogenesis of moyamoya vasculopathy. ${ }^{2,13,15-17,19,23}$ In this study, we found that unilateral MMD may be more likely to be associated with autoimmune disease than bilateral MMD. Moreover, these findings suggest that unilateral MMD may be more frequently observed with autoimmune comorbidities. Moyamoya disease presenting concurrently with other specific autoimmune diseases has also been described in the literature. . $^{2,9,13,16,19,23}$ In this study, autoimmune thyroid diseases, all other autoimmune diseases, and other comorbidities tended to have higher prevalence rates in unilateral MMD patients than in bilateral MMD patients. Nevertheless, no significant differences were observed between unilateral and bilateral MMD with regard to each specific concurrent autoimmune disease, including GD, HT, Type 1 diabetes mellitus, and all other evaluated autoimmune diseases. Strikingly, however, the overall autoimmune disease prevalence was significantly higher in the unilateral MMD patients than in the bilateral MMD patients. These results indicate that not only is unilateral MMD associated with autoimmune disease, but bilateral MMD is as well, a conclusion that is consistent with findings of multiple previous studies. , $13,15-17,19,23^{-1}$
More importantly, we found that unilateral MMD was more likely associated with autoimmune disease than bilateral MMD. Different pathogenetic mechanisms may underlie moyamoya vessel formation in unilateral and bilateral MMD. Our findings augmented the hypothesis that unilateral MMD is a more common underlying condition than bilateral MMD. However, MMD pathogenesis and the correlation between MMD and autoimmune disease has to be further elucidated. Previous reports suggested that T-cell dysregulation and special immunological stimulation conditions (e.g., autoimmune thyroid disease) may relate to cellular proliferation and vascular dysregulation in MMD. ${ }^{13,16,20}$ Several studies have suggested that an association exists between MMD and autoimmune diseases, including Type 1 diabetes mellitus, AIG, SLE, APAS, APS, PBC, GD, and HT. ${ }^{2,7,15,17,18,20,22,23,25}$ Furthermore, several reports have demonstrated that thyroid autoantibodies may be associated with the moyamoya development..$^{13,15,16}$ Our study also indicated that autoimmune abnormalities may be associated with pathophysiological mechanisms of moyamoya vasculopathy formation.

There were some limitations to our study. First, we used the ICD-9 code to identify patients; therefore, a selection bias could have occurred if this code was incorrectly applied. This was a retrospective study, but we tried our best to ensure accuracy with regard to the clinical diagnoses and unilateral and bilateral MMD identification. We acknowledge that the selection bias that often occurs in retrospective studies may also influence the interpretation of results. Second, our study was limited to patients from a single hospital, which may limit its generalizability to other patient populations. However, this study raised the importance of evaluating MMD because of the higher autoimmune disease prevalence that we observed in unilateral than in bilateral MMD.

\section{Conclusions}

This study found a higher overall autoimmune disease prevalence in patients with unilateral MMD than in those with bilateral MMD. Unilateral MMD may be associated with autoimmune disease to a higher degree than bilateral MMD, and autoimmune comorbidities may be more frequently observed in patients with unilateral disease. Different pathogenetic mechanisms may underlie moyamoya vessel formation in unilateral and bilateral MMD. Establishing relevant management guidelines for these 2 subgroups is necessary.

\section{Acknowledgments}

This study was supported by grants from the Doctoral Fund of Ministry of Education of China (20080441210) and Special Foundation of Sichuan Postdoctor (2009064).

\section{References}

1. Ahn IM, Park DH, Hann HJ, Kim KH, Kim HJ, Ahn HS: Incidence, prevalence, and survival of moyamoya disease in Korea: a nationwide, population-based study. Stroke 45:1090-1095, 2014

2. Bower RS, Mallory GW, Nwojo M, Kudva YC, Flemming KD, Meyer FB: Moyamoya disease in a primarily white, 
Midwestern US population: increased prevalence of autoimmune disease. Stroke 44:1997-1999, 2013

3. Duan L, Bao XY, Yang WZ, Shi WC, Li DS, Zhang ZS, et al: Moyamoya disease in China: its clinical features and outcomes. Stroke 43:56-60, 2012

4. Fukui M: Guidelines for the diagnosis and treatment of spontaneous occlusion of the circle of Willis ('moyamoya' disease). Research Committee on Spontaneous Occlusion of the Circle of Willis (Moyamoya Disease) of the Ministry of Health and Welfare, Japan. Clin Neurol Neurosurg 99 (Suppl 2):S238-S240, 1997

5. Hayashi K, Horie N, Izumo T, Nagata I: A nationwide survey on unilateral moyamoya disease in Japan. Clin Neurol Neurosurg 124:1-5, 2014

6. Hayashi K, Horie N, Suyama K, Nagata I: An epidemiological survey of moyamoya disease, unilateral moyamoya disease and quasi-moyamoya disease in Japan. Clin Neurol Neurosurg 115:930-933, 2013

7. Houkin K, Abe H, Yoshimoto T, Takahashi A: Is "unilateral" moyamoya disease different from moyamoya disease? J Neurosurg 85:772-776, 1996

8. Jiang T, Perry A, Dacey RG Jr, Zipfel GJ, Derdeyn CP: Intracranial atherosclerotic disease associated with moyamoya collateral formation: histopathological findings. J Neurosurg 118:1030-1034, 2013

9. Kainth DS, Chaudhry SA, Kainth HS, Suri FK, Qureshi AI: Prevalence and characteristics of concurrent down syndrome in patients with moyamoya disease. Neurosurgery 72:210215,2013

10. Kazumata K, Ito M, Tokairin K, Ito Y, Houkin K, Nakayama $\mathrm{N}$, et al: The frequency of postoperative stroke in moyamoya disease following combined revascularization: a single-university series and systematic review. J Neurosurg 121:432440, 2014

11. Kelly ME, Bell-Stephens TE, Marks MP, Do HM, Steinberg GK: Progression of unilateral moyamoya disease: A clinical series. Cerebrovasc Dis 22:109-115, 2006

12. Kim JM, Jung KH, Sohn CH, Park J, Moon J, Han MH, et al: High-resolution MR technique can distinguish moyamoya disease from atherosclerotic occlusion. Neurology 80:775776, 2013

13. Kim SJ, Heo KG, Shin HY, Bang OY, Kim GM, Chung CS, et al: Association of thyroid autoantibodies with moyamoyatype cerebrovascular disease: a prospective study. Stroke 41:173-176, 2010

14. Kuroda S, Houkin K: Moyamoya disease: current concepts and future perspectives. Lancet Neurol 7:1056-1066, 2008

15. Leno C, Mateo I, Cid C, Berciano J, Sedano C: Autoimmunity in Down's syndrome: another possible mechanism of moyamoya disease. Stroke 29:868-869, 1998

16. Li H, Zhang ZS, Dong ZN, Ma MJ, Yang WZ, Han C, et al:
Increased thyroid function and elevated thyroid autoantibodies in pediatric patients with moyamoya disease: a case-control study. Stroke 42:1138-1139, 2011

17. Sasaki T, Nogawa S, Amano T: Co-morbidity of moyamoya disease with Graves' disease. Report of three cases and a review of the literature. Intern Med 45:649-653, 2006

18. Scott RM, Smith ER: Moyamoya disease and moyamoya syndrome. N Engl J Med 360:1226-1237, 2009

19. Suzuki S, Mitsuyama T, Horiba A, Fukushima S, Hashimoto N, Kawamata T: Moyamoya disease complicated by Graves' disease and type 2 diabetes mellitus: report of two cases. Clin Neurol Neurosurg 113:325-329, 2011

20. Tendler BE, Shoukri K, Malchoff C, MacGillivray D, Duckrow R, Talmadge T, et al: Concurrence of Graves' disease and dysplastic cerebral blood vessels of the moyamoya variety. Thyroid 7:625-629, 1997

21. Uchino K, Johnston SC, Becker KJ, Tirschwell DL: Moyamoya disease in Washington State and California. Neurology 65:956-958, 2005

22. Utku U, Asil T, Celik Y, Tucer D: Reversible MR angiographic findings in a patient with autoimmune Graves disease. AJNR Am J Neuroradiol 25:1541-1543, 2004

23. Wang R, Xu Y, Lv R, Chen J: Systemic lupus erythematosus associated with moyamoya syndrome: a case report and literature review. Lupus 22:629-633, 2013

24. Watanabe C, Oishi T, Yamamoto T, Sasaki K, Tosaka M, Sato T, et al: Chorea and Broca aphasia induced by diabetic ketoacidosis in a type 1 diabetic patient diagnosed as moyamoya disease. Diabetes Res Clin Pract 67:180-185, 2005

\section{Disclosure}

The authors report no conflict of interest concerning the materials or methods used in this study or the findings specified in this paper.

\section{Author Contributions}

Conception and design: You, Chen. Acquisition of data: Chen, Liu, Sun, He. Analysis and interpretation of data: Chen, Liu, Zhou, Sun, He. Reviewing the vascular images: You, Chen, Liu. Drafting the article: Chen. Critically revising the article: You, Chen, Zhou. Reviewed submitted version of manuscript: Chen. Statistical analysis: Chen. Study supervision: You, Liu, Zhou.

\section{Correspondence}

Chao You, Department of Neurosurgery, West China Hospital, West China School of Medicine, Sichuan University, No. 37, Guoxue Alley, Chengdu 610041, Sichuan Province, People's Republic of China. email: huaxisw@163.com. 